\title{
Selective hypersensitivity to a single nonsteroidal anti-inflammatory drug
}

\author{
Helena Klar ${ }^{1}$, Nika Sotošek ${ }^{1}$, Julij Šelb ${ }^{1}$, Mitja Košnik ${ }^{1,2 \bowtie}$
}

\begin{abstract}
Introduction: The aim of the study was to explore the frequency and the clinical and diagnostic characteristics of a single nonsteroidal anti-inflammatory drug-induced hypersensitivity (SNIUAA) subgroup.

Methods: In the hospital information system we identified patients who performed the oral provocation test (OPT) with analgesics between 2004 and 2016. We divided patients into phenotype groups based on clinical history and OPT results.

Results: Altogether, 248 patients (44.3\% of phenotyped patients) were diagnosed with SNIUAA. The most common offending drug in SNIUAA was diclofenac $(52.4 \%)$, followed by pyrazolones $(30.7 \%)$. In the group with selective diclofenac hypersensitivity, there was a larger percentage $(40.0 \%)$ of patients with anaphylaxis. Compared to other phenotypes, significantly fewer patients had OPT performed with the suspected drug in SINUAA; however, in $69.4 \%$ of patients in this group, OPT with acetylsalicylic acid was performed.

Conclusions: SNIUAA was the most common phenotype of nonsteroidal anti-inflammatory drug hypersensitivity in our cohort. Diclofenac was the most common cause of the hypersensitivity reaction and also the most common cause of anaphylactic reaction. In the group of patients with a positive history after taking diclofenac or pyrazolone, fewer OPTs with the suspected drug were performed, but more often OPT with aspirin to prove that hypersensitivity is selective.
\end{abstract}

Keywords: NSAID hypersensitivity, oral provocation test, diclofenac, pyrazolones, anaphylaxis

Received: 10 April 2019| Returned for modification: 26 June 2019| Accepted: 18 July 2019

\section{Introduction}

Nonsteroidal anti-inflammatory drugs (NSAIDs) have been reported to be among the most common drugs causing adverse reactions (1). They are related to 21 to $25 \%$ of all adverse drug reactions (2). The majority of adverse effects result from the drugs' pharmacological activity; these include stomach pain, gastrointestinal bleeding, and tinnitus. These adverse effects are dose-related and predictable, and can be seen in any treated patient, especially if a patient takes a higher cumulative dose. Hypersensitivity reactions are rarer and only occur in susceptible individuals in response to a small dose of the drug (3). NSAIDs are the second most common cause of hypersensitivity reactions, after beta-lactam antibiotics (4). The prevalence of NSAID hypersensitivity in the general population is between $0.6 \%$ and $2.5 \%$, and is higher among patients with asthma (between $4.6 \%$ and $11.0 \%$ ) (5). Moreover, NSAIDs are the cause of more than $50 \%$ of drug-induced anaphylaxis (6). Hypersensitivity reactions can be divided into non-allergic or cross-reactive and allergic or selective. Cross-reactivity results from inhibition of COX-1 enzyme and involves reactions to several chemically non-related NSAIDs (3). Selective hypersensitivity occurs when a patient reacts only to a single NSAID or different NSAIDs with a very similar chemical structure (3). Selective hypersensitivity can be divided into two types: single NSAID-induced urticaria/angioedema/anaphylaxis (SNIUAA) and NSAIDs-induced delayed hypersensitivity reactions (NIDHR) (3).

Patients with SNIUAA usually develop symptoms within a few minutes or 1 hour after a NSAID consumption. The symptoms range from mild urticaria and localized angioedema to anaphylaxis. In SNIUAA, the clinical spectrum of symptoms and timing of reactions suggest an allergic type I mechanism (3).

The aim of this study was to define the NSAID hypersensitivity patterns in patients referred to a Slovenian drug allergy clinic and to explore the clinical and diagnostic characteristics of the SNIUAA subgroup.

\section{Methods}

Patients

The Golnik University Clinic of Respiratory and Allergic Diseases is the only center in Slovenia performing diagnostics in adult patients with a suspicion of NSAID hypersensitivity. In the hospital information system we identified patients who had been subjected to an oral provocation test (OPT) with any analgesic between 2004 and 2016, and we reviewed their medical records.

We selected a group of patients with a firm diagnosis of NSAID hypersensitivity. They had either a positive provocation test or a convincing medical history of hypersensitivity reactions (Fig. 1). We evaluated the probability of NSAID hypersensitivity based on the data in the patients' medical records as follows: i) low probability of hypersensitivity: spontaneous occurrence of urticaria also during the time when analgesics were not used, or a medical history of simultaneous infection and fever; ii) moderate probability of hypersensitivity: non-specific symptoms coincided with the use of analgesic; the patient did not fulfil the criteria for high pre-test probability; or iii) high probability of hypersensitivity: reaction developed less than 4 hours after the intake of the first dose of analgesic and manifested with urticaria, angioedema, nasal stuffiness, dyspnea, or anaphylaxis, which could not be explained 
with any other reason; or positive OPT.

Medical files were first reviewed by junior physicians (HK, NS). In cases of uncertainty, the medical record was discussed in a panel with a senior physician (MK). If the data in the medical record were contradictory or insufficient, the patients were classified as moderate probability and excluded from phenotyping.

\section{Diagnostic procedure}

The vast majority of patients underwent a provocation test. In the case of a low pre-test probability, we proceeded with a single blind four-step gradual OPT with the culprit drug starting with 1/100 of the full dose, continuing after 90 minutes with $1 / 10$, half, and full dose. The protocol was adapted from EAACI/GA2LEN guidelines (5). In patients with a history of aspirin hypersensitivity, reactions after multiple NSAIDs or aspirin-induced asthma, we performed OPT with COX-2 inhibitors or paracetamol. In patients with a history of anaphylaxis after NSAID, we started with a gradual aspirin challenge.

\section{Phenotyping of hypersensitivity reactions}

We divided patients into five groups based on the clinical phenotype of hypersensitivity reaction observed in clinical history according to the ENDA task force classification (3):

- NERD: NSAIDs-exacerbated respiratory disease. Dyspnea and/or rhinitis after NSAID intake;

- NECD: NSAIDs-exacerbated cutaneous disease. Deterioration of chronic urticaria after NSAID intake;

- NIUA: NSAIDs-induced urticaria/angioedema. Urticaria and/ or angioedema after NSAID intake;

- SNIUAA: single NSAID-induced urticaria/angioedema/anaphylaxis;

- NIDHR: NSAIDs-induced delayed hypersensitivity reactions.

\section{Statistical analysis}

The data are presented as the average and standard deviation. We used a $t$-test and chi-square test to observe the difference between the groups and results were considered statistically significant if the $p$-value was less than 0.05. All statistical analyses were performed using Microsoft Excel and GraphPad Prism 5 (GraphPad Software, La Jolla, CA, USA) software.

\section{Results}

Between 2004 and 2016, provocation tests with analgesics were performed in 2,090 patients (69.1\% female) (Table 1$)$. A total of 1,489 OPTs were performed with culprit analgesics, and 144 of these were positive. Overall, the provocation test was positive in $224(10.7 \%)$ patients. There were no statistically significant differences in age between the groups of patients with positive and negative test results $(47.1 \pm 14.6$ years vs. $47.8 \pm 16.2$ years; $p>0.05$, $t$-test). The female-to-male ratio in the group of patients with positive test results was 1:1.54, and the ratio in the group with negative test results was 1:2.35 ( $p<0.05$, chi square test).

\begin{tabular}{lcc}
\multicolumn{3}{l}{ Table 1 | Frequency of tests with analgesics. } \\
\hline Analgesic & Tests performed & Positive \\
\hline Aspirin & 950 & $146(15.4 \%)$ \\
Paracetamol & 866 & $37(4.3 \%)$ \\
Meloxicam & 419 & $12(2.9 \%)$ \\
Naproxen & 333 & $20(6.0 \%)$ \\
Tramadol & 187 & $3(1.6 \%)$ \\
Diclofenac & 129 & $6(4.7 \%)$ \\
Ketoprofen & 112 & $5(4.5 \%)$ \\
Coxib & 70 & $3(4.3 \%)$ \\
Ibuprofen & 46 & $2(4.3 \%)$ \\
Pyrazolone & 8 & $2(25.0 \%)$ \\
Etodolac & 6 & $1(16.7 \%)$ \\
\hline Total & 3,126 & $237(7.6 \%)$ \\
\hline
\end{tabular}

\section{Phenotyping of hypersensitivity reactions}

For phenotyping hypersensitivity reactions, a total of 559 patients with a clear diagnosis of hypersensitivity (Table 2) were included: 224 patients with positive provocation tests (133 female, 91 male, age $46.9 \pm 14.7$ years) and 335 patients with a convincing medical history of hypersensitivity reaction but without OPT performed with the culprit drug (236 female, 99 male, age $51.9 \pm 14.8$ years). There was a statistically significant difference in the female-to-

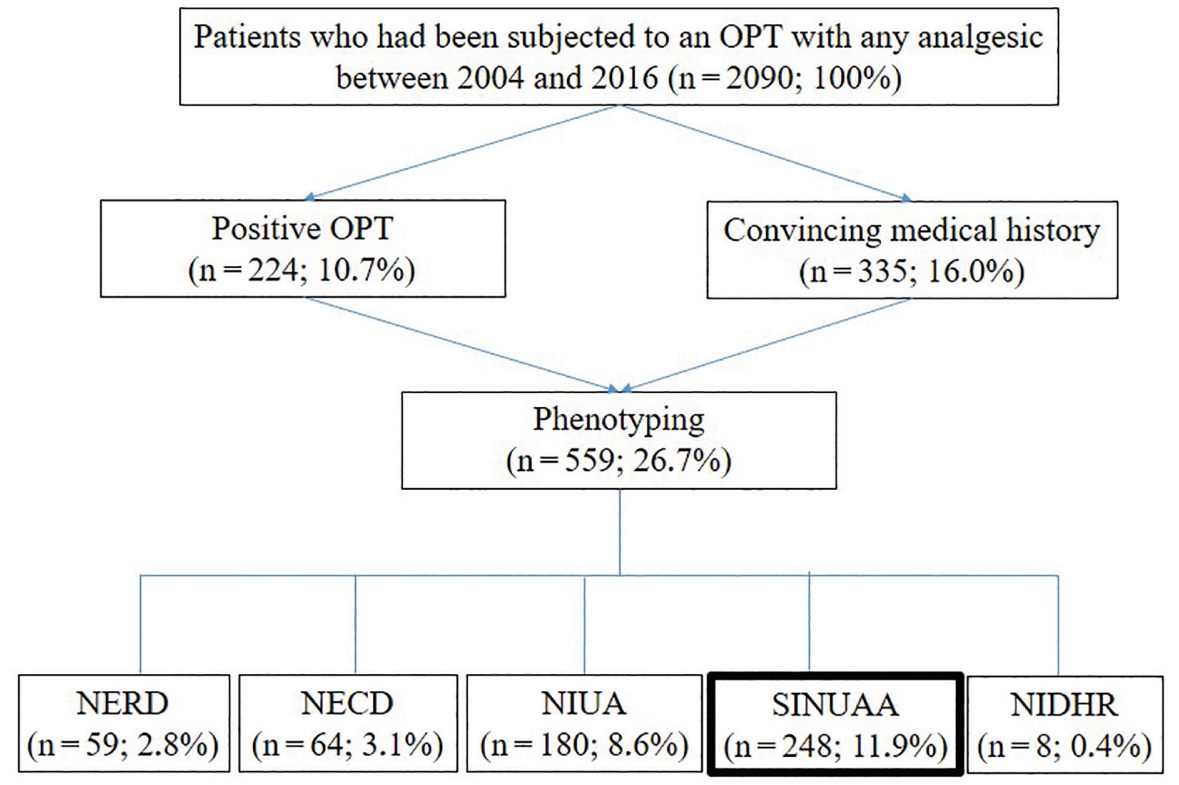

Figure 1 | Flow chart showing inclusion of patients in the study.

$\mathrm{OPT}=$ oral provocation test; NERD = NSAIDs-exacerbated respiratory disease; NECD = NSAIDs-exacerbated cutaneous disease; NIUA = NSAIDs-induced urticaria/ angioedema; SNIUAA = single NSAID-induced urticaria/angioedema/anaphylaxis; NIDHR = NSAIDs-induced delayed hypersensitivity reactions. 
male ratio ( $p<0.05$, chi square test) and age ( $p<0.05, t$-test) between the two subgroups of patients.

\section{Phenotype SNIUAA}

A total of 248 patients were classified as SNIUAA phenotype, which represents $44.3 \%$ of all patients who had their hypersensitivity reaction phenotype analyzed (Table 2). In Table 3 we present the frequency of hypersensitivity to different analgesics in the subgroup of patients with a SNIUAA phenotype. Diclofenac accounted for $52.4 \%$ and pyrazolones for $30.7 \%$ of patients in the SNIUAA phenotype. Selective hypersensitivity in patients with a Table 2 | Clinical phenotype of reaction after analgesic intake.

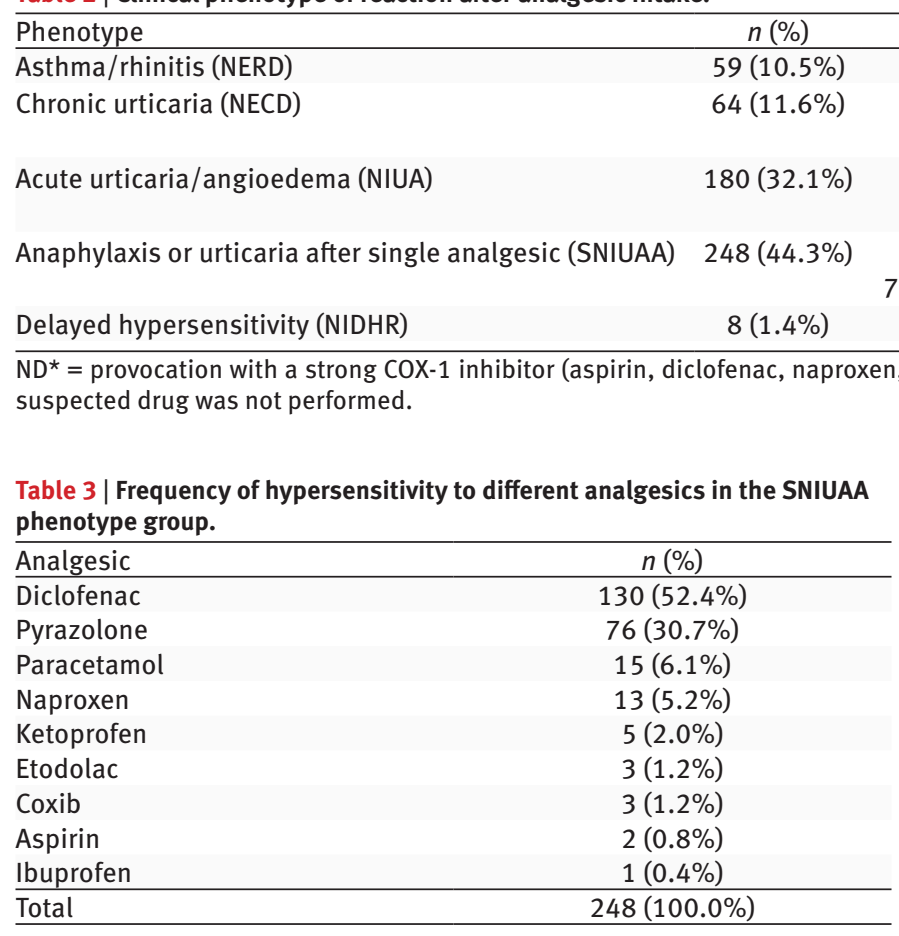

clinical history of having a reaction after pyrazolone and sodium diclofenac intake was mostly diagnosed based on a high pretest probability of a reaction after those two analgesics and negative OPT result with aspirin (Table 4).

Table 5 presents the clinical spectrum of symptoms observed in patients with a selective hypersensitivity. Most patients present with urticaria/angioedema, followed by anaphylaxis and asthma exacerbation.

Table 6 presents the number of analgesics prescribed in Slovenia in 2017 (7). Aspirin is by far the most commonly prescribed drug (including aspirin $100 \mathrm{mg}$ used as an antiplatelet drug), followed by naproxen and diclofenac.
Table 4 | Diagnosis of selective hypersensitivity to single analgesics in the SNIUAA phenotype group.

Table 6 | Analgesic prescription in Slovenia in 2017. Slovenian health care insurance data (7).

\begin{tabular}{lc}
\hline Drug & DDD, $n$ \\
\hline Aspirin & $52,824,250$ \\
Naproxen & $12,564,080$ \\
Diclofenac & $10,416,197$ \\
Paracetamol & $5,046,293$ \\
Ibuprofen & $3,135,926$ \\
Coxib & $2,042,754$ \\
Pyrazolone & $1,804,516$ \\
Tramadol & $1,350,672$ \\
Meloxicam & $1,170,140$ \\
Ketoprofen & $1,076,018$ \\
Etodolac & 958,290 \\
Combination of tramadol and paracetamol & $6,567,030$
\end{tabular}

\begin{tabular}{|c|c|c|c|c|}
\hline & \multirow[b]{2}{*}{$\begin{array}{l}\text { Diagnosis based on positive OPT with } \\
\text { suspected analgesic: patients, } n(\%)\end{array}$} & \multicolumn{2}{|c|}{ Diagnosis based on high clinical probability: patients, $n(\%)$} & \multirow[b]{2}{*}{ Total } \\
\hline & & Negative OPT with aspirin & $\begin{array}{c}\text { Diagnosis based on convincing } \\
\text { clinical presentation }\end{array}$ & \\
\hline Diclofenac & $7(5.4 \%)$ & $100(76.9 \%)$ & $23(17.7 \%)$ & 130 \\
\hline Pyrazolone & $4(5.3 \%)$ & $62(81.6 \%)$ & $10(13.2 \%)$ & 76 \\
\hline Paracetamol & $13(86.7 \%)$ & $2(13.3 \%)$ & 0 & 15 \\
\hline Naproxen & $7(53.9 \%)$ & $4(30.8 \%)$ & $2(15.4 \%)$ & 13 \\
\hline Ketoprofen & $1(20.0 \%)$ & $3(60.0 \%)$ & $1(20.0 \%)$ & 5 \\
\hline Etodolac & $1(33.3 \%)$ & $1(33.3 \%)$ & $1(33.3 \%)$ & 3 \\
\hline Coxib & $3(100.0 \%)$ & 0 & 0 & 3 \\
\hline Aspirin & $1(50.0 \%)$ & 0 & $1(50.0 \%)$ & 2 \\
\hline Ibuprofen & 0 & 0 & $1(100.0 \%)$ & 1 \\
\hline Total & $37(14.9 \%)$ & $172(69.4 \%)$ & $39(15.7 \%)$ & $248(100.0 \%)$ \\
\hline
\end{tabular}

OPT $=$ oral provocation test.

Table 5 | Clinical presentation in patients with a selective hypersensitivity.

\begin{tabular}{|c|c|c|c|c|c|}
\hline \multirow{2}{*}{ Drug } & \multicolumn{5}{|c|}{ Patients, $n(\%)$} \\
\hline & Urticaria/ngioedema & Anaphylaxis & Asthma exacerbation & Other* & Total \\
\hline Diclofenac & $77(59.2 \%)$ & $52(40.0 \%)$ & 0 & $1(0.8 \%)$ & 130 \\
\hline Pyrazolone & $55(72.4 \%)$ & $18(23.7 \%)$ & $2(2.6 \%)$ & $1(1.3 \%)$ & 76 \\
\hline Paracetamol & $10(66.7 \%)$ & $4(26.7 \%)$ & $1(6.7 \%)$ & 0 & 15 \\
\hline Naproxen & $10(76.9 \%)$ & $2(15.4 \%)$ & $1(7.7 \%)$ & 0 & 13 \\
\hline Ketoprofen & $5(100.0 \%)$ & 0 & 0 & 0 & 5 \\
\hline Etodolac & $2(66.7 \%)$ & $1(33.3 \%)$ & 0 & 0 & 3 \\
\hline Coxib & $3(100.0 \%)$ & 0 & 0 & 0 & 3 \\
\hline Aspirin & 0 & $1(50.0 \%)$ & 0 & $1(50.0 \%)$ & 2 \\
\hline Ibuprofen & $1(100.0 \%)$ & 0 & 0 & 0 & 1 \\
\hline Total & $163(65.7 \%)$ & $78(31.5 \%)$ & $4(1.6 \%)$ & $3(1.2 \%)$ & 248 \\
\hline
\end{tabular}

*Other: pruritus of palms, feet, or scalp. 


\section{Discussion}

We have shown that selective NSAID hypersensitivity was the most frequent phenotype (44.3\%) in patients diagnosed with NSAID hypersensitivity at the adult drug allergy clinic. The most common causes of selective hypersensitivity were diclofenac (52.4\%) and pyrazolones (30.7\%).

Despite the high prevalence of hypersensitivity to NSAID and the importance of determining the phenotypes, there is very little literature on this subject to enable comprehensive comparison of our data. A study by Pérez-Alzate et al. showed that in some countries cross-hypersensitivity is more common whereas in others selective hypersensitivity dominates (8). In our study, among 559 patients whose diagnosis of hypersensitivity to analgesic was certain, $44.3 \%$ patients had anaphylaxis or urticaria after a single analgesic. The observed frequency of selective hypersensitivity to analgesic was similar to that in the study performed by PérezAlzate (8). However, it is possible that patients with anaphylaxis, which is a common clinical presentation of SNIUAA, seek medical advice more often than patients with a delayed rash, rhinitis, acute urticaria, or other minor reactions which might skew the results and lead to overestimation of SNIUAA.

The Spanish study refers to pyrazolones (9) as the main reason for selective hypersensitivity; in contrast, our study suggests that diclofenac is the most common culprit drug in SNIUAA (52.4\%). Pyrazolones rank second, at $30.7 \%$. There might be a concern that the epidemiology of hyper-reactivity reaction might simply follow the proportions of NSAIDs prescribed. However, we have shown that diclofenac ranked only third among the NSAIDs prescribed (7). On the other hand, diclofenac is prescribed six times more often compared to pyrazolones, and so we can speculate that pyrazolones are much more allergenic compared to diclofenac. Whereas records are available for prescribed analgesics only, paracetamol, aspirin, ibuprofen, and naproxen (but not diclofenac and pyrazolones) can also be bought over-the-counter. Thus the usage of diclofenac and pyrazolones is not underestimated.

A study by Picaud et al. found that diclofenac is strongly associated with anaphylaxis (10). Our conclusions were similar, with $40 \%$ of diclofenac-allergic patients presenting with anaphylaxis. Anaphylaxis was less frequent in patients with selective hypersensitivity to pyrazolones (23.4\%), paracetamol (26.7\%), and naproxen $(15.4 \%)$.

Many studies have shown that a diagnostic procedure in patients with SNIUAA should begin with aspirin OPT $(2,11,12)$. This procedure enables separation of the cross-reactors from patients with selective hypersensitivity and prevent patients from unnecessarily avoiding a large group of analgesics. Ariza et al. showed that the selective hypersensitivity diagnosis for pyrazolones is most often based on convincing clinical history and negative oral aspirin provocation testing (9), which is in agreement with our results. In our study, the SNIUAA group contained the most patients who did not have an OPT with the suspected analgesic; however, in almost $80 \%$ of patients with selective diclofenac or pyrazolone hypersensitivity an aspirin OPT was performed.

Even if a patient reports having a reaction after different NSAIDs, especially if the reaction is anaphylaxis, it is appropriate to test tolerance of aspirin - Pérez-Alzate et al. have shown that $7.9 \%$ of patients with selective hypersensitivity were hypersensitive to two or even more NSAIDs from different chemical groups (8).

Previous studies have shown that approximately $40 \%$ of pa- tients with selective hypersensitivity to metamyzole have a positive cutaneous test or basophil activation test (8). Patients with selective hypersensitivity for other NSAIDs usually have negative cutaneous tests despite the fact the reaction resembles IgE-mediated allergy. In our study, cutaneous tests were not routinely performed as a part of diagnostic workup.

Several articles about patients with hypersensitivity to NSAID can be found in the literature. Pérez-Alzate et al. included 697 patients with confirmed NSAID hypersensitivity, 203 of them with SNIUAA (8). An Italian study included 159 patients with NSAID hypersensitivity, but only those with urticarial or angioedema reaction (12), and Danish studies included 147 patients with a reaction after ingestion of NSAID (13). Our cohort is relatively large compared to other studies, with 2,090 patients included who had an OPT between 2004 and 2016. However, only 559 patients $(26.8 \%)$ were included in the phenotype analysis because in the rest of the patients NSAID hypersensitivity was excluded or was unlikely. When the medical history is unconvincing, a provocation test is performed with the aim of excluding drug hypersensitivity. When the clinical history is convincing, we are confronted with an ethical dilemma whether to start the diagnostic procedure with a provocation test with the culprit drug, which would probably elicit a hypersensitivity reaction, or to instead perform a provocation test with an alternative drug with the aim of showing good tolerance. We showed that in real-life clinical practice the diagnostic procedure is more often focused on determining the analgesic that the patient can safely receive.

The research has some advantages and disadvantages. It was carried out as a retrospective analysis of the data generated during clinical work. In the cohort there are many patients; however, because several different physicians were involved in the diagnostics, it is likely that the diagnostic procedure was not always the same. Moreover, the diagnostic approach has also changed over the years. In addition, we did not consider the possibilities of observer and recall biases, which could significantly impact result interpretation. A concern might be raised that the selection of patients for the study by screening for patients who underwent a provocation test with analgesics might miss patients with a history of very severe reactions or very high index of suspicion in their history who were not challenged by a culprit drug. However, even patients with a high index of suspicion normally undergo a provocation test to confirm good tolerance with at least one alternative analgesic, such as paracetamol or selective COX-2 inhibitors. Lastly, we also did not perform follow-up visits to check for the tolerability of alternative NSAIDs, which SNIUAA patients were told they can safely receive.

\section{Conclusions}

An important finding of our study is that a large proportion of NSAID-intolerant patients do not tolerate only a single drug. A history of a reaction after pyrazolones and diclofenac, particularly anaphylaxis, is a strong predictor that the patient belongs to the SNIUAA group.

\section{Acknowledgements}

The authors thank the Allergology Department staff for performing the clinical work that was necessary to obtain the data for the analysis and Sara Marinček for reading the text. 


\section{Statement of ethics}

This study was approved by a state ethics committee (study no. 0120-647/2015-2), and patients provided informed consent for using anonymized data in the analysis.

\section{References}

1. Bennett PN, Brown MJ, Sharma P. Clinical pharmacology. 11th ed. Edinburgh: Churchill Livingstone; 2012

2. Malskat WSJ, van der Tas C, Knulst AC, Bruijnzeel-Koomen CAFM, Röckmann H. Aspirin tolerance in patients with NSAID hypersensitivity. Allergy. 2010;1197-8.

3. Kowalski ML, Makowska JS. Seven steps to the diagnosis of NSAIDs hypersensitivity: how to apply a new classification in real practice? Allergy Asthma Immunol Res. 2015;7:312-20.

4. Rodriguez-Trabado A, Camara-Hijon C, Ramos-Cantarino A, Porcel-Carreno SL, Jimenez-Timon S, Pereira-Navarro G, et al. Basophil activation test for the in vitro diagnosis of nonsteroidal anti-inflammatory drug hypersensitivity. Allergy Asthma Proc. 2008;29:241-9.

6. Nizankowska-Mogilnicka E, Bochenek G, Mastalerz L, Świerczyńska M, Picado C, Scadding G, et al. EAACl/GA2LEN guideline: aspirin provocation tests for diagnosis of aspirin hypersensitivity. Allergy. 2007;62:1111-8.

6. Jares EJ, Baena-Cagnani CE, Sanchez-Borges M, Ensina LF, Arias-Cruz A, Gómez $M$, et al. Drug-induced anaphylaxis in Latin American countries. J Allergy Clin Immunol Pract. 2015;3:780-8.

7. Zavod za zdravstveno zavarovanje Slovenije. Podatki o porabi zdravil [Internet]. [cited 2019 Jan 11]. Available from: https://partner.zzzs.si/wps/portal/portali/ aizv/zdravila_in_zivila_za_posebne_zdravstvene namene/podatki_o_porabi_zdravil/!ut/p/z1/04_Sj9CPykssyoxPLMnMzovMAflj08ziTQxdPd2N_Q08LSyCDQocjZzMzXz8XQosTAzoC7IdFQGdnpEx/. Slovenian.

\section{Funding sources}

The research was supported by the Grant P3-0360 from the Slovenian Research Agency.

8. Pérez-Alzate D, Cornejo-García JA, Pérez-Sánchez N, Andreu I, García-Moral A Agúndez JA, et al. Immediate reactions to more than one NSAID must not be considered as cross-hypersensitivity unless ASA tolerance is verified. J Investig Allergol Clin Immunol. 2017;27:32-9.

9. Ariza A, Garcia-Martin E, Salas M, Montañez MI, Mayorga C, Blanca-Lopez N, et al. Pyrazolones metabolites are relevant for identifying selective anaphylaxis to metamizole. Sci Rep. 2016;23845.

10. Picaud J, Beaudouin E, Renaudin JM, Pirson F, Metz-Favre C, Dron-Gonzalvez M, et al. Anaphylaxis to diclofenac: nine cases reported to the Allergy Vigilance Network in France. Allergy. 2014;69:1420-3.

11. Asero R. Oral aspirin challenges in patients with a history of intolerance to single non-steroidal anti-inflammatory drugs. Clin Exp Allergy. 2005;35:713-6.

12. Zisa G, Riccobono F, Bommarito L, D’Antonio C, Calamari AM, Poppa M, et al. Provocation tests with the offending nonsteroidal anti-inflammatory drugs in patients with urticaria/angioedema reactions. Allergy Asthma Proc. 2012;33: 421-6.

13. Nissen CV, Bindslev-Jensen C, Mortz CG. Hypersensitivity to non-steroidal antiinflammatory drugs (NSAIDs): classification of a Danish patient cohort according to EAACI/ENDA guidelines. Clin Transl Allergy. 2015;5:10. 